\title{
Line-Shape Transition of Collision Broadened Lines
}

\author{
H. Harde, ${ }^{1}$ N. Katzenellenbogen, ${ }^{2}$ and D. Grischkowsky ${ }^{2}$ \\ ${ }^{1}$ Universität der Bundeswehr Hamburg, Holstenhofweg 85, 22043 Hamburg, Germany \\ ${ }^{2}$ Oklahoma State University, Stillwater, Oklahoma 74078
}

(Received 19 July 1994)

\begin{abstract}
Using the newly developed technique of $\mathrm{THz}$ time-domain spectroscopy, we have measured the farwing absorption line profile of the ensemble of collision broadened ground state rotational lines of methylchloride vapor out to more than 200 linewidths from resonance, corresponding to frequency offsets as much as $5 \times$ the resonant frequency. On these far wings the measured absorption is approximately an order of magnitude less than that predicted by the van Vleck-Weisskopf theory. Our observations show that at higher frequencies a transition occurs from the regime of the van VleckWeisskopf theory to the regime of the Lorentz theory.
\end{abstract}

PACS numbers: $32.70 . J z$

The study of molecular collisions has led to a relatively complete understanding of collision broadening phenomena in gases [1-3]. Important information on the collision behavior and intermolecular forces is obtained through the width and shape of broadened spectral lines from optical to microwave frequencies. For the higher frequency range of optical and infrared transitions, the simple Lorentzian line shape [4] provides excellent agreement with experiment, particularly for the central region of the line. As the frequencies are reduced to those of the microwave or far-infrared region, and under conditions when a linewidth becomes comparable with the transition frequency, the absorption profile of a spectral line is better represented by a van Vleck-Weisskopf line shape [5-8].

The line-shape theory of Lorentz [4,5] assumes that after a collision the molecules are oriented randomly with respect to the applied electromagnetic field. Later, it was shown by van Vleck and Weisskopf [5] that this assumption prevents the Lorentz theory from reducing to that of Debye for the zero resonant frequency case. This discrepancy was removed by the van Vleck-Weisskopf theory based on the assumption that after a collision the molecules are oriented with a Boltzmann distribution along the applied electromagnetic field [1,5]. This assumption required that the duration of the collision be short compared to the period of oscillation of the applied field.

In the $\mathrm{THz}$ experiments reported here, we measured the absorption line shapes in the ground state rotational band of methylchloride vapor from relatively near resonance to frequency offsets as much as $5 \times$ the resonant frequency and to as much as 200 linewidths away. These experimental conditions are unprecedented. Our measurements are well fit using a van Vleck-Weisskopf line shape for an individual transition out to frequencies of the order of $1 \mathrm{THz}$. Further, increasing the frequency leads to a much reduced absorption compared to that of the van Vleck-Weisskopf line shape. In fact, we find that at higher frequencies the measured absorption is almost an order of magnitude less than that given by the van VleckWeisskopf line shape.

We have observed, for the first time, the switching between the van Vleck-Weisskopf and Lorentz collision theories. Our observations are well explained by the following picture $[1,5]$. At lower frequencies our measurements satisfy the condition that the collision duration is short compared to the period of oscillation of the applied field. However, as the frequency is further increased, the above condition is violated and the original approximation of Lorentz becomes applicable; the molecules become randomly oriented with respect to the applied field after a collision. We obtain an excellent fit to our measurements by using the van Vleck-Weisskopf theory for relatively low frequencies and with a weighting function switching to the Lorentz theory for the high frequency part.

For our investigations we used the powerful new technique of $\mathrm{THz}$ time-domain spectroscopy (TDS) [915]. With this technique two electromagnetic pulse shapes are measured, the input (reference) pulse and the propagated pulse, which has changed shape due to its passage through the sample under study. Consequently, via Fourier analyses of the input and propagated pulses, the frequency dependent absorption and dispersion of the sample can be obtained. Because of the broad bandwidth of the $\mathrm{THz}$ pulses, a spectral range extending from low frequencies up to more than $5 \mathrm{THz}$ can be investigated.

The high-performance optoelectronic setup used to generate and detect the subpsec pulses of freely propagating $\mathrm{THz}$ electromagnetic radiation is shown in Fig. 1 and has been described elsewhere [9-11]. A GaAs transmitting antenna with simple coplanar transmission line geometry [11] and a silicon on sapphire receiving antenna consisting of a micron size dipole antenna imbedded in a coplanar transmission line were both optoelectronically driven by 65 fs pulses from a colliding-pulse mode-locked dye laser. The generated pulses of $\mathrm{THz}$ radiation are collimated by a silicon lens attached to the back of the trans- 


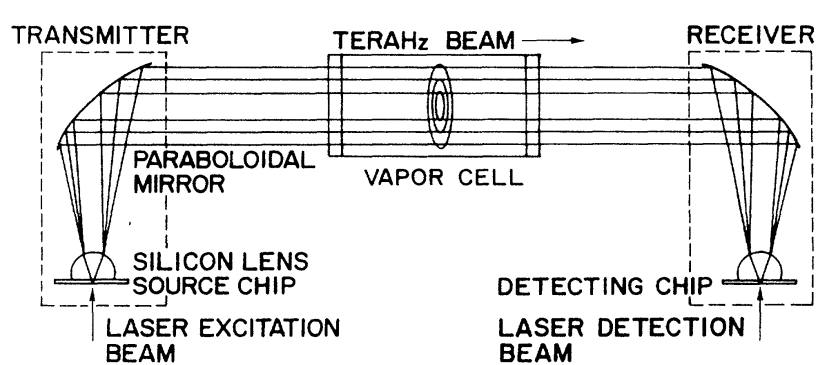

FIG. 1. THz time domain spectroscopy setup.

mitting chip and directed onto a paraboloidal mirror that recollimates the radiation into a beam which propagates through the sample cell and is then focused by an identical mirror and lens set onto the receiving antenna. The timedependent voltage induced across the receiving antenna is determined by measuring the collected charge (current) versus the time delay between the laser excitation and detection (gating) pulses.

A $\mathrm{THz}$ pulse measured after passage through an empty sample cell is displayed in Fig. 2(a), illustrating the rapid response of the system, the clean baseline, and the excellent signal-to-noise ratio. The THz pulse of Fig. 2(a) is shown again on an expanded time scale in Fig. 2(b), where the time delay from the maximum to minimum is only $0.35 \mathrm{ps}$. The corresponding amplitude spectrum, obtained by a numerical Fourier transform of Fig. 2(a), is displayed as the upper curve in Fig. 2(c), together with the calculated absorption spectrum for the ground state rotational band of methylchloride at a pressure of $1000 \mathrm{hPa}$ and for the $22.5 \mathrm{~cm}$ sample length. This band is completely overlapped by the spectrum of the excitation pulse. At frequencies higher than $2 \mathrm{THz}$ the absorption is almost exclusively determined by the far wings of the rotational lines.

When the sample cell is filled with $1000 \mathrm{hPa}$ of methylchloride vapor, as shown in Fig. 3(a), the reshaped and attenuated excitation pulse is followed by a series of coherent transients [13-15], which rapidly decay because of the strong collisional broadening. At the lower pressure of $10 \mathrm{hPa}$ the coherent transients extend to beyond $400 \mathrm{ps}$ [15]. The reshaped excitation pulse of Fig. 3(a) is displayed on an expanded time scale in Fig. 3(b), where the dramatic reshaping can be seen in detail. The time shift of the pulse compared to Fig. 2(a) is due to the index of refraction of the vapor. The numerical Fourier transform of Fig. 3(a) is displayed as the lower curve in Fig. 3(c), where it is compared to the incident spectrum derived from the Fourier transform of the $\mathrm{THz}$ pulse of Fig. 2(a). Although the central portion of this spectrum has been almost completely absorbed by the vapor, the absorption rapidly decreases on the far wings of the lines and becomes imperceptible beyond 3.5 THz.

Quantitative evaluation of these data requires the simulation of the transmitted absorption spectrum shown in
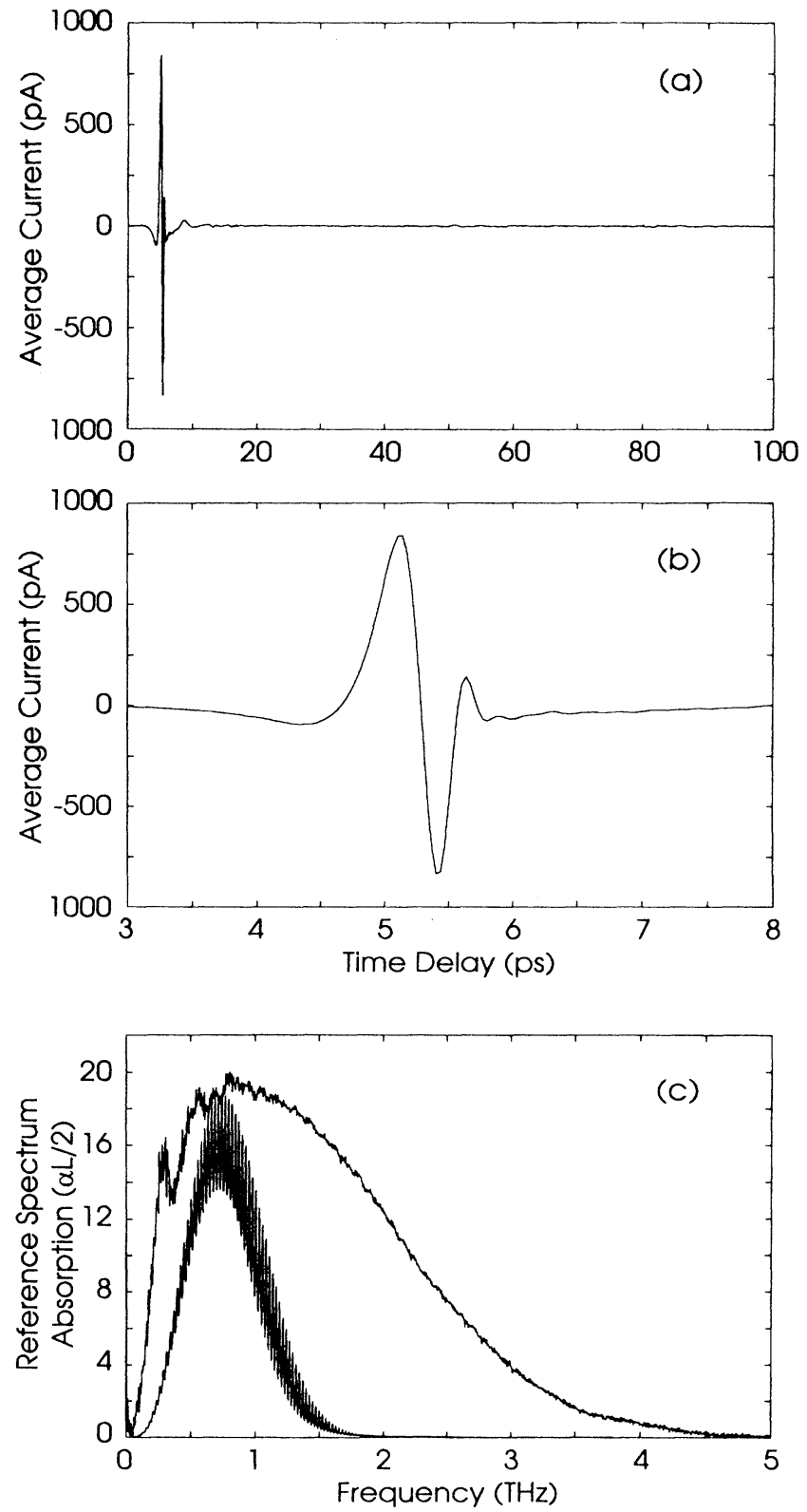

FIG. 2. (a) Measured $\mathrm{THz}$ reference pulse transmitted through an empty vapor cell. (b) Measured $\mathrm{THz}$ reference pulse on an expanded time scale. (c) Amplitude spectrum (upper curve) of the reference pulse compared to the calculated methylchloride absorption spectrum.

Fig. 3(c), obtained by multiplying the incident spectrum with the theoretical absorption of the rotational band. An analysis directly in the time domain is also possible when the complex rotational spectrum (consisting of the absorption and dispersion) multiplied by the input spectrum is transformed back to the time domain and the simulated pulse structure is compared with Fig. 3(a). 

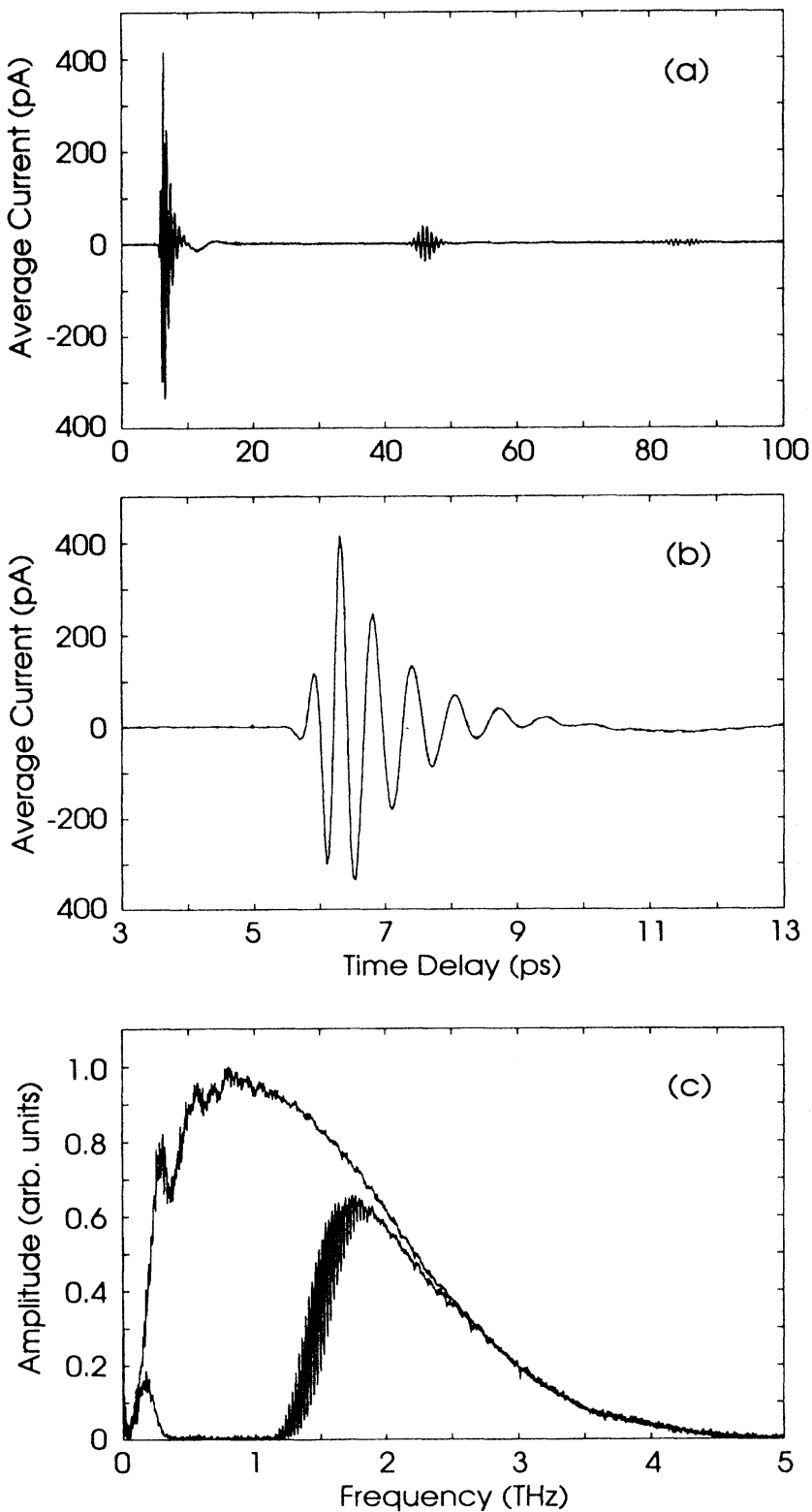

FIG. 3. (a) Measured and calculated transmitted $\mathrm{THz}$ pulses for $1000 \mathrm{hPa}$ methylchloride vapor. (b) Measured and calculated $\mathrm{THz}$ pulses on an expanded time scale. (c) Amplitude spectrum (upper curve) of reference pulse compared to spectrum of $1000 \mathrm{hPa}$ transmitted pulse.

The absorption and dispersion over the rotational band is found by calculating these for each individual line using the known molecular constants and summing over all transitions within the considered spectral range. We assume a simple superposition of the line-shape functions of the van Vleck-Weisskopf and the Lorentz theories of the form

$$
\begin{aligned}
\alpha_{J K}(\omega)=C_{J K} \omega^{2}[ & \frac{\Delta \omega_{J K} f_{S}^{+}\left(\omega, \omega_{J K}\right)}{\left(\omega-\omega_{J K}\right)^{2}+\left(\Delta \omega_{J K} / 2\right)^{2}} \\
& \left.+\frac{\Delta \omega_{J K} f_{S}^{-}\left(\omega, \omega_{J K}\right)}{\left(\omega+\omega_{J K}\right)^{2}+\left(\Delta \omega_{J K} / 2\right)^{2}}\right],
\end{aligned}
$$

for the power absorption coefficient, for each relevant transition from $J, K \rightarrow J+l, K$ ( $J$ is the rotational quantum number and $K$ is the projection quantum number). The phase shift per unit length $\Delta k_{J K}(\omega)$ is given by

$$
\begin{aligned}
& \Delta k_{J K}(\omega)=2 C_{J K} \frac{\omega_{J K}^{2} \omega}{\omega_{J K}^{2}-\omega^{2}}\left[1-\frac{\omega \Delta \omega_{J K}^{2}}{8 \omega_{J K}^{2}}\right. \\
& \times\left(\frac{\left(\omega+\omega_{J K}\right) f_{S}^{+}\left(\omega, \omega_{J K}\right)}{\left(\omega-\omega_{J K}\right)^{2}+\left(\Delta \omega_{J K} / 2\right)^{2}}\right. \\
&\left.\left.\quad+\frac{\left(\omega-\omega_{J K}\right) f_{S}^{-}\left(\omega, \omega_{J K}\right)}{\left(\omega+\omega_{J K}\right)^{2}+\left(\Delta \omega_{J K} / 2\right)^{2}}\right)\right],
\end{aligned}
$$

where $\omega_{J K}$ is the transition angular frequency, $\Delta \omega_{J K}=$ $2 / \tau_{J K}$ is the angular frequency linewidth (FWHM) with $\tau_{J K}$ as the mean time between collisions, and $C_{J K}$ is a transition dependent proportionality factor determined by the dipole matrix element, the pressure, temperature, and the Boltzmann distribution (see Ref. [15]). The experimental data are well fitted by an emperically derived switching function controlling the transition from the van Vleck-Weisskopf line shape at low frequencies to the Lorentz theory line shape at higher frequencies; this function is given by

$$
f_{S}^{ \pm}\left(\omega, \omega_{J K}\right)=\frac{1 \pm \omega \omega_{J K} \tau_{c}^{2}}{1+\omega^{2} \tau_{c}^{2}},
$$

where $\tau_{c}$ is the collision parameter. The above Eq. (1) for $\alpha$ is equivalent to $\alpha=A \alpha_{\mathrm{vVW}}+B \alpha_{\mathrm{LT}}$, where the absorption coefficient $\alpha_{\mathrm{vVw}}$ of van Vleck-Weisskopf theory [Eq. (17) of Ref. [5]] is obtained for $\omega \tau_{c} \ll 1$ and $\alpha_{\mathrm{LT}}$ of Lorentz theory [Eq. (9) of Ref. [5]] for $1 \ll \omega \tau_{c} ; A+B=1$, and they are given by $A=1 /[1+$ $\left.\left(\omega \tau_{c}\right)^{2}\right]$ and $B=\left(\omega \tau_{c}\right)^{2} /\left[1+\left(\omega \tau_{c}\right)^{2}\right]$. It is noted that in either the $\alpha_{\mathrm{vVW}}$ or the $\alpha_{\mathrm{LT}}$ limit, the line shape is determined by the mean time between collisions $\tau_{J K}$, only the switching function involves the collision parameter $\tau_{c}$.

As long as the collision parameter is short compared to the oscillation period of the $\mathrm{THz}$ radiation field (adiabatic hypothesis of Ref. [5]), directly after a collision the dipoles can be assumed to be in thermal equilibrium with the radiation field, i.e., their orientations are distributed in accordance with the Boltzmann law. This orientation to the field is correlated with some small absorption of the field even far away from any molecular resonance. It determines the constant absorption on the far wing of the van Vleck-Weisskopf line shape. However, if the oscillations of the field are faster than $\tau_{c}$, no thermal equilibrium can be established and the van Vleck-Weisskopf 
theory converges to the Lorentz theory, for which it is assumed that after each collision the mean distribution of the dipoles is completely random and unpolarized.

It is tempting to associate the collision parameter $\tau_{c}$ with the duration of the molecular collision. However, a few words of caution are in order. First the mean collision duration time, estimated from the collision cross section and thermal velocity, is expected to be of the order of 1 ps. Second, the simple assumption of treating the absorption and dispersion as a sum of that of the two theories, with the relative weighting controlled by a switching function, can only be a first approximation to a more complete theory for this intermediate frequency regime. Here, the functional form of the switching function has been determined emperically and assumes an exponential behavior in the time domain for the molecular orientation process, thus giving Eq. (3) in the frequency domain. However, if a step function with a width $\tau_{c}$ for the molecular orientation process were assumed, the frequency $\nu$ would replace the angular frequency $\omega$ in Eq. (3). Third the wing absorption of van Vleck-Weisskopf theory is particularly sensitive to state changing (diabatic) collisions causing any reorientation of the molecules to the external field [5], while the full linewidth of a rotational line is additionally determined by phase changing (adiabatic) collisions. Since any alignment of molecules during a collision is expected to be much faster than the average overall collision duration time, we propose to interprete $\tau_{c}$ as a measure of the orientation time of molecules due to a collision.

Independent of the above cautionary discussion, simulations based on the superposition theory give excellent agreement with the measurement in the frequency domain as well as in the time domain. The actual amplitude absorption $\alpha(\omega) L / 2$, where $\alpha(\omega)$ is the power absorption coefficient and $L$ is the cell length, is obtained from the data of Fig. 3(c). The absorption is shown for the high frequency wing in Fig. 4(c) (bottom curve) where it is compared to that calculated from superposition theory using a collision parameter of $\tau_{c}=200 \mathrm{fs}$. On this scale the measurement and calculation agree so well that they appear as a single line, while the absorption based on the van Vleck-Weisskopf profile (upper curve) is almost an order of magnitude too large. Also the calculated (from superposition theory) pulse structure, sensitive to the absorption as well as to the dispersion, agrees so well with the measured transmitted excitation pulse and first coherent transient that it is indistinguishable when plotted together, as is shown in Figs. 3(a) and 3(b). Variation of

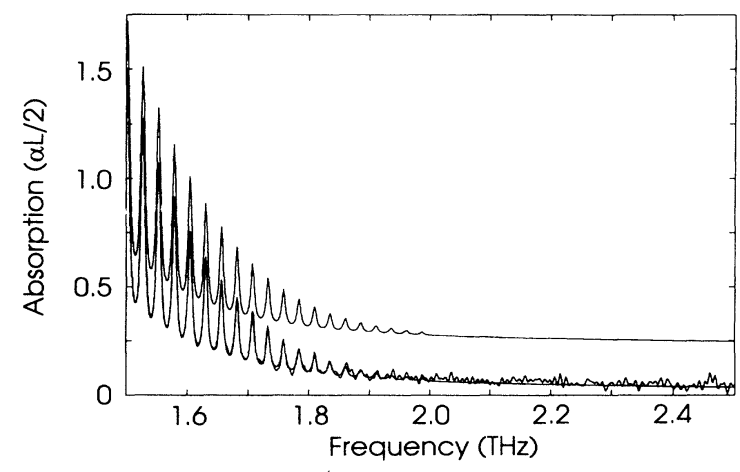

FIG. 4. Experimentally determined absorption of the $1000 \mathrm{hPa}, 22.5 \mathrm{~cm}$ long methylchloride vapor [obtained from Fig. 3(c)] compared to that calculated for the van VleckWeisskopf line shape (upper curve) and for the superposition theory (lower curve).

the fitting parameters indicate an uncertainty of $30 \%$ for the collision parameter.

[1] C. H. Townes and A.L. Schawlow, Microwave Spectroscopy (Dover Publications, Inc., New York, 1975).

[2] J. R. Fuhr, L. J. Rozman, and W. L. Wiese, Bibliography on Atomic Line Shapes and Shifts, NBS Special Publication No. 366 (U.S. GPO, Washington, DC, 1974).

[3] I. I. Sobelman, L. A. Vainshtein, and E. A. Yukov, Excitation of Atoms and Broadening of Spectral Lines, Springer Series: Chemical Physics 7 (Springer-Verlag, Heidelberg, 1981).

[4] H. A. Lorentz, Proc. Amst. Akad. Sci. 8, 591 (1906).

[5] J.H. Van Vleck and V.F. Weisskopf, Rev. Mod. Phys. 17, 227 (1945).

[6] H. Fröhlich, Nature (London) 157, 478 (1946).

[7] R. Karplus and J. Schwinger, Phys. Rev. 73, 1020 (1948).

[8] P. W. Anderson, Phys. Rev. 76, 647 (1949).

[9] Ch. Fattinger and D. Grischkowsky, Appl. Phys. Lett. 54, 490 (1989).

[10] M. van Exter and D. Grischkowsky, IEEE Trans. Microwave Theory Tech. 38, 1684 (1990).

[11] N. Katzenellenbogen and D. Grischkowsky, Appl. Phys. Lett. 58, 222 (1991).

[12] D. Grischkowsky, S. Keiding, M. van Exter, and Ch. Fattinger, J. Opt. Soc. Am. B 7, 2006 (1990).

[13] H. Harde, S. Keiding, and D. Grischkowsky, Phys. Rev. Lett. 66, 1834 (1991).

[14] D. Harde and D. Grischkowsky, J. Opt. Soc. Am. B 8, 1642 (1991).

[15] H. Harde, N. Katzenellenbogen, and D. Grischkowsky, J. Opt. Soc. Am. B 11, 1018 (1994). 